\title{
Article
}

\section{Parafoveal processing across different lexical constituents in Chinese reading}

Cui, L., Drieghe, Denis, Yan, G., Bai, X., Chi, H. and Liversedge, Simon Paul

Available at http://clok.uclan.ac.uk/22389/

Cui, L., Drieghe, Denis, Yan, G., Bai, X., Chi, H. and Liversedge, Simon Paul ORCID: 0000-0002-8579-8546 (2013) Parafoveal processing across different lexical constituents in Chinese reading. The Quarterly Journal of Experimental Psychology Section B, 66 (2). pp. 403-416. ISSN 1747-0218

It is advisable to refer to the publisher's version if you intend to cite from the work. http://dx.doi.org/10.1080/17470218.2012.720265

For more information about UCLan's research in this area go to http://www.uclan.ac.uk/researchgroups/ and search for <name of research Group>.

For information about Research generally at UCLan please go to http://www.uclan.ac.uk/research/

All outputs in CLoK are protected by Intellectual Property Rights law, including Copyright law. Copyright, IPR and Moral Rights for the works on this site are retained by the individual authors and/or other copyright owners. Terms and conditions for use of this material are defined in the policies page.

\section{CLoK}

Central Lancashire online Knowledge www.clok.uclan.ac.uk

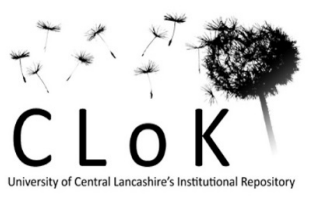


Parafoveal processing across different lexical constituents in Chinese reading

Lei Cui

Tianjin Normal University, China

Jining University, Shandong, China

Denis Drieghe

University of Southampton, UK

Guoli Yan, Xuejun Bai, Hui Chi

Tianjin Normal University, China

Simon P. Liversedge

University of Southampton, UK

Corresponding Author:

Simon P. Liversedge,

Centre for Visual Cognition,

School of Psychology,

Shackleton Building,

University of Southampton,

Highfield, Southampton, SO17 1BJ, UK

Email: s.p.liversedge@soton.ac.uk

Phone: +44 2380599399

Fax: +442380594597

Running Head: Chinese reading 


\begin{abstract}
We report a boundary paradigm eye movement experiment to investigate whether the linguistic category of a two character Chinese string affects how the second character of that string is processed in the parafovea during reading. We obtained clear preview effects in all conditions, but more importantly, found parafoveal-on-foveal effects whereby a nonsense preview of the second character influenced fixations on the first character. This effect occurred for monomorphemic words, but not for compound words or phrases. Also, in a word boundary demarcation experiment, we demonstrate that Chinese readers are not always consistent in their judgments of which characters in a sentence comprise words. We conclude that information regarding the combinatorial properties of characters in Chinese is used on-line to moderate the extent to which parafoveal characters are processed.
\end{abstract}


The extent to which we pre-process text in the parafovea during reading has become an extremely important issue in recent years due to contrasting accounts of processing provided by the two leading models of oculomotor control during reading. The E-Z Reader model (e.g., Reichle, Pollatsek, Fisher, \& Rayner, 1998; Reichle, Rayner, \& Pollatsek, 2003) posits that attention is shifted serially and sequentially like a spotlight, with the words of the sentence being lexically processed one by one. In contrast, the SWIFT model (e.g., Engbert, Nuthmann, Richter, \& Kliegl, 2005) specifies that two or more words can be lexically processed (and potentially identified) in parallel. An important point to note is that the vast majority of studies that have investigated whether words are processed serially, or in parallel during reading have focused on effects between words that are sequential in the text. If it can be demonstrated that the lexical properties of word $n+1$ affect processing while word $n$ is fixated, then a parafoveal-on-foveal effect is said to have occurred (Kennedy, 2000; Murray, 1998; see Drieghe, 2011 and Schotter, Angele, \& Rayner, 2011 for recent reviews). Such effects are fundamentally damaging to serial models of processing, but are entirely consistent with parallel models.

Many studies that have investigated whether words are processed serially or in parallel have employed the boundary paradigm (Rayner, 1975). In the boundary paradigm an invisible boundary is inserted in a sentence, and the text to the right of that boundary is changed prior to its fixation. Before the reader crosses the boundary with their eyes, the parafoveal words are systematically manipulated relative to the words that appear at fixation after a saccade crosses the boundary. 
Thus, when the text is in the parafovea it appears different during preview than when it is fixated. Using this experimental technique, it is possible to determine which characteristics of parafoveal text were extracted and processed prior to fixation. The extent to which an identity preview facilitates processing relative to an experimental preview is referred to as the preview benefit (Rayner, 1975; Rayner, 1982; 1998).

Although traditionally parafoveal-on-foveal effects have been examined between adjacent words in a sentence, more recently there have been a number of experiments employing the boundary paradigm within words to investigate whether the constituents of morphologically complex words (e.g., compound words like basketball) are processed serially or in parallel (e.g., Drieghe, Pollatsek, Juhasz, \& Rayner, 2010; Hyönä, Bertram, \& Pollatsek, 2004; Juhasz, Pollatsek, Hyönä, Drieghe, \& Rayner, 2009). That is to say, these experiments have investigated serial or parallel lexical processing of linguistic constituents within a word rather than between words.

Hyönä et al. (2004) used the boundary paradigm to investigate processing of Finnish sentences containing 12- to 18-letter compound words. The boundary was located between the first and second constituent of the compound, and the preview of the second constituent was manipulated (being a visually dissimilar letter string, or an identity string). Very substantive within word parafoveal preview effects (101 ms) were obtained, and Hyönä et al. noted that this finding stands in stark contrast to other studies that have examined preview effects between words. Such studies have shown effects ranging between 5 and $30 \mathrm{~ms}$, with the average being approximately 14 
ms. Hyönä et al. argued that the much larger parafoveal preview effects they obtained may have occurred for two reasons: First, the eyes were closer to the preview due to the lack of a space between the constituents. Secondly, the second constituent is part of a single linguistic unit (the compound word), which may result in the second constituent capturing attention earlier than would be the case if it is was a separate word. A key point to note in relation to the Hyönä et al. study is that it was the first in which eye-contingent display changes were made within words.

More recently, Juhasz et al. (2009) directly tested the effect of spacing on preview effects in compound words. They used spaced compound (e.g., tennis ball) and unspaced compound (e.g., basketball) words as targets embedded in sentences. Spaced and unspaced compounds are single linguistic units, however, the space between constituents in spaced compounds ensures that the preview of the second constituent is one space away from the fixated word. Consistent with Hyönä et al., Juhasz et al. obtained greater preview benefit for unspaced than spaced words. To extend these findings, Juhasz et al. carried out a follow-up experiment in which they used spaced compounds and adjective-noun pairs, for which there are no single corresponding lexical entries within the readers' mental lexicon. In their second experiment, there was a numerically larger preview effect for spaced compounds (31 ms) than for adjective-noun pairs $(20 \mathrm{~ms})$, however, this difference did not attain statistical significance. Juhasz et al. argued that the effects for the spaced compounds could be due to them comprising a single lexical unit, and the similar effects for adjective noun pairs could be due to the noun being syntactically 
predictable from the adjective.

The most recent study that is directly related to the current experiment was conducted by Drieghe et al. (2010) who also assessed the effect of morphological structure on parafoveal processing whilst controlling for visual acuity effects on parafoveal information. Drieghe et al. investigated differences in parafoveal processing between monomorphemic (e.g., fountain) and unspaced compound words (e.g. bathroom). In their study, Drieghe et al. used previews that preserved the first two letters of the second lexeme and substituted random letters for the remainder in the compound word, with correspondingly similar previews in the counterpart monomorphemic word. They found a larger preview effect for gaze duration on the monomorphemic word $(225 \mathrm{~ms})$ than on the compound word $(123 \mathrm{~ms})$. Drieghe et al. also obtained a significant parafoveal-on-foveal effect for the monomorphemic words. This effect was absent when the preview of the corresponding letters in the second constituent of the unspaced compound was incorrect. These results indicate that the morphological structure of a word does modulate the extent to which a word part is processed in the parafovea. When the word is monomorphemic, it appears that the second portion of the word is processed earlier and to a greater degree in the parafovea than when the word is morphologically complex.

From the studies discussed above, we can safely conclude that in spaced alphabetic languages, both word spacing and the morphological structure of a word influence how it is parafoveally processed before it is fixated. However, not all languages are word spaced and alphabetic. A good example of such a language is 
Chinese. Chinese is a logographic script wherein the written text is formed from strings of equally spaced box-like symbols called characters. Chinese characters are themselves comprised of sub-parts termed radicals that can represent aspects of a word's meaning or phonology. Although a Chinese character can in principle contain quite a number of radicals, most characters contain one, two or three. The radicals themselves are comprised of strokes, which are features like dots, dashes and lines, and these are the smallest units of visual information within a character. Thus, characters comprised of strokes and radicals are the basic units of visual information in the written form of Chinese. Note, however, that characters can (though need not necessarily) combine to form words. Most Chinese words with more than one character are comprised of two characters, though a small number of words contain three or more characters. Furthermore, some strings of characters represent phrases or well-known idioms that often form meaningful units of linguistic information. Thus, although the basic unit of information in Chinese is the character, it is certainly the case that there are linguistically meaningful representations associated with words or phrases that are comprised of multiple character units. Another point of note concerns the fact that in Chinese there is far greater ambiguity as to word boundaries than is the case in spaced alphabetic languages such as English. Thus, although most Chinese readers agree on the characters that comprise most Chinese words, it is also the case that not all Chinese readers agree on the characters that comprise all words. Clearly, a lack of word spacing to demarcate word identities in written Chinese contributes to this ambiguity and disagreement. 
Finally, the lack of spaces between words in Chinese means that the written form of the language is quite dense (i.e., reduced in its horizontal spatial extent), much more so than more spatially extended languages such as English, or Finnish which contains many long compound words. This means that parafoveal linguistic information is closer to the point of fixation in more than less dense languages. It is not surprising, therefore, that many studies have shown stronger and earlier preview effects in Chinese reading than in English reading (Wang, Tong, Yang, \& Leng, 2009; Yan, Richter, Shu, \& Kliegl, 2009; Yang, Wang, Xu, \& Rayner, 2009; Yen, Radach, Tzeng, Hung, \& Tsai, 2009). Thus, Chinese has three important properties that make it an extremely interesting language in which to consider how readers carry out morphological processing of words when they fall both in foveal and parafoveal vision. First, there are no overt word boundaries (spaces) demarking the beginning and ending of words. Secondly, there can be ambiguity about the characters that comprise words. Thirdly, the language is visually dense, thereby providing optimal conditions in which to observe effects of parafoveal information on foveal processing.

For these reasons, we carried out a boundary paradigm eye movement experiment that was quite similar to that reported by Drieghe et al., to investigate preview effects between the constituent characters of different kinds of words or phrases in Chinese. We constructed identical sentence frames into which we embedded three types of two-character target words: (1) Compound words comprised of two characters where each character corresponds to a word, and the two words correspond directly to the overall meaning of the whole compound (e.g., 灯塔 means beacon in English; the 
first character means lamp, and the second means tower). (2) Monomorphemic words for which the meaning of each individual character did not directly correspond to the overall meaning of the target word (e.g., 玫瑰 means rose in English, but each character alone has no meaning in Chinese). (3) Adjective-noun word pairs where each word was comprised of a single character and the two characters together did not form a single word (e.g., 斜塔 means leaning tower in English).

Similar to Drieghe et al. (2010), we were specifically interested to investigate how readers processed the second character of our target words prior to their direct fixation, and therefore, we positioned the boundary between the first and the second character of our target words. We used either identity or nonsense character previews. In this way, we were able to determine whether the nature of the first (foveal) character of a target character pair influenced the extent to which readers preprocessed the second (parafoveal) character for each type of target character pair we used. On the basis of Drieghe et al. we predicted greater preview effects for monomorphemic words than for compound word or adjective-noun pairs. We included the adjective-noun condition to allow us to evaluate whether we might obtain differences in preview when the target was two compared with one word. Although we had no direct experimental basis on which to generate predictions, we anticipated that if any effect did occur, it would be greater for compound words than for phrases since compound words are single linguistic units, whereas adjective-noun pairs are not. Finally, our experimental conditions also allowed us to investigate whether parafoveal-on-foveal effects occurred (i.e., an effect of the nonsense preview on 
fixations on the first character prior to the boundary change), and whether these were modulated by the nature of the target character pair.

\section{Method}

Subjects. Fifty-two undergraduates from Tianjin Normal University were given a small present to participate in the experiment. They were all native speakers of Chinese with normal or corrected-to-normal vision.

Apparatus. Eye movements were collected using a SR Research Eyelink 2000 eye tracker that monitored the position of the right eye every ms. The sentences were presented in simple Song font in black on a white background. Each character was approximately $2.1 \times 2.1 \mathrm{~cm}^{2}$ in size. The viewing distance of the subject to the screen was $60 \mathrm{~cm}$. At this distance, each character subtended approximately $2^{\circ}$ of visual angle, thereby maximizing the possibility that the target word was located in the parafovea when the pre-target word was fixated (Balota \& Rayner, 1991).

Materials and Design. The design was a 3 (Target Type: monomorphemic word, compound word, phrase) $\times 2$ (Preview Type: identical and dissimilar preview) within subject design. A set of 60 monomorphemic words, compound words and phrase triplets were identified. Within each triplet, the compound word and phrase contained the same second constituent, and the first characters of each were matched on the number of strokes and frequency. The selection of the tightly matched compound words and phrases imposed constraints on the monomorphemic words that could be selected. Despite this, efforts were made to minimize differences in stroke 
complexity of the first and second characters across the triplet, and differences in word frequency in relation to the compound word of the triplet (see Table 1). The word frequency was not different between monomorphemic and compound words, $t(59)=1.15, p=.26$. There were significant differences for the first character of the triplet on the number of strokes $\left(F(2,177)=11.84, M S_{e}=89.84, p<.001\right)$ and frequency $\left(F(2,177)=4.84, M S_{e}=850,807, p<.001\right)$. The mean number of strokes for the first character of the monomorphemic word was significantly larger than the mean number for the compound word and the phrase $(p \mathrm{~s}<.01)$. The first character frequency of the monomorphemic word was significant smaller than that of the compound word and the phrase $(p \mathrm{~s}<.05)$. The first character of the compound word and phrase were matched on their mean number of strokes and frequency $(p s>.10)$. The mean number of strokes for the second character of the monomorphemic word was significantly larger than that of the compound word and phrase $(t(59)=4.62, p$ $<.01)$. The second character frequency of the monomorphemic word was significantly lower than that of compound word and phrase $(t(59)=3.14, p<.01)$. Note that the second character of the compound word and the phrase stimuli was the same).

Insert Table 1 about here

The dissimilar previews were pseudocharacters created using True Font software. They very closely resembled real characters but were completely meaningless, being 
comprised of inappropriate radical combinations (though the number of radicals was matched with the target as closely as possible). Furthermore, the pseudocharacter previews did not contain any of the radicals (semantic or phonetic) of the target character (see Cui, Yan, Bai, Hyönä, Wang \& Liversedge, 2012).

Sentence frames were created for each target word or phrase triplet such that besides the target character pair itself, the content was identical up until the word after the target. After this point differences could occur, but these were minimal and all the sentences made sense. The verb before the target characters was a two character compound. The sentences appeared on just one line with a maximum of seventeen characters. For all sentences the target string appeared towards the middle of the line of text.

The invisible boundary was placed between the two characters of the target. As soon as the eyes moved across the invisible boundary, the preview character was replaced by the target character (the display change took approximately $13 \mathrm{~ms}$ to complete).

An example sentence pair is given in Figure 1.

Insert Figure 1 about here

\section{Rating of materials.}

Second character predictability. A list of sentence fragments including all of the characters prior to the second of the target characters was given on a sheet of paper. 
Twenty-four subjects that did not take part in the eye movement experiment (but were otherwise comparable) were asked to provide the next character such that the sentence could be continued meaningfully. The target type was counterbalanced across three files using a Latin square design such that each experimental file contained each sentence frame only once. Eight subjects were assigned to each experimental file. There was significant difference for the predictability of the second character of the target word or phrase, $F_{2}(2,118)=144.38, M S_{e}=4.44, p<.001$. The predictability was higher for the monomorphemic word $(M=7.05, S D=1.66)$ than the compound word $(M=1.67, S D=2.31 ; t(59)=12.78, p<.001)$ and the phrase $(M=1.15, S D=$ $1.88 ; t(59)=17.61, p<.001)$. There was no significant difference between the compound word and the phrase, $t<1.32$. These findings are entirely consistent with our expectations, given that the first characters of the monomorphemic words most often appear with their second characters, whilst this is far less the case for the compound words and the phrases.

Plausibility rating. A plausibility pretest was conducted to ensure that each of the target character pairs in a triplet fitted equally well in the sentence frames. Thirty subjects (again, different to those that took part in the eye movement experiment) were asked to rate the target sentences for their plausibility, using a 5-point scale $(1=$ very plausible, $5=$ very implausible). Besides the 60 experimental sentences, we included 20 additional sentences that were a little implausible (to allow for potential for range in ratings). There were no significant differences in plausibility ratings between monomorphemic words $(M=2.06, S D=0.33)$, compound words $(M=1.96$, 
$S D=0.27)$ and phrases $(M=2.01, S D=0.34), F<1$.

Procedure. Subjects were given written instructions for the experiment prior to its start, and the experimenter then went through these with the subject verbally, answering any questions. Next, a 3-point calibration was performed. Calibration accuracy was checked before the presentation of each sentence and a re-calibration performed whenever necessary. Subjects were told to read sentences for comprehension at their own rate. The items were counterbalanced using a Latin square design such that the subjects saw each target character pair only once. In total, subjects read 96 sentences: 60 experimental sentences randomly intermingled with 30 fillers sentences, preceded by 6 practice sentences. After every three sentences, a comprehension question was asked about the preceding sentence. The subjects answered the questions by pressing a Yes or No key on a gamepad. After the experiment, subjects were asked whether they experienced anything unusual during reading. A small number of subjects reported seeing something flicker on the screen on only one or two trials. No subject was able to report exactly what it was that they had seen. Including 5 minutes for the initial calibration of the eye-tracking system, the whole experiment lasted about 25 minutes.

\section{Results}

Four subjects were discarded because their comprehension accuracy was below 75\% (Rayner, 1998). For the 48 subjects included in the analyses, the mean comprehension accuracy was $91.3 \%$. Trials on which the display change occurred during a fixation due to drift or microsaccades were excluded. Fixations less than 60 
ms, or greater than $600 \mathrm{~ms}$ were also excluded. In total $9.8 \%$ of the data was excluded (including track losses). We carried out repeated measures Target Type (monomorphemic word; compound word; phrase) $\times$ Preview Type (identical; dissimilar) ANOVAs on subjects and items means for each of our measures.

Eye fixation measures for the first constituent

We first considered fixation durations on the first constituent (see Table 2) as these may potentially reflect parafoveal-on-foveal effects.

Insert Table 2 about here

First Fixation Duration. For the first fixation duration, the main effect of preview type did not attain significance, $F_{1}(1,47)=2.87, M S_{e}=1,134, p=.09 ; F_{2}(1,59)=2.61$, $M S_{e}=1,553, p=.11$. Numerically, however, the first fixation durations for the dissimilar previews were longer $(238 \mathrm{~ms})$ than for the identical previews $(231 \mathrm{~ms})$. There was no significant main effect of target type $\left(F_{\mathrm{S}}<1.5\right)$, indicating that first fixations of the first character of the target character pair were similar for the different target types.

There was, however, a significant target type by preview type interaction $\left(F_{1}(2,94)=3.54, M S_{e}=1,005, p<.05 ; F_{2}(2,118)=6.03, M S_{e}=1,538, p<.01\right)$. For monomorphemic words there was a significant difference $(17 \mathrm{~ms})$ between identical and dissimilar preview conditions, $\left(t_{1}(47)=2.60, p<.05 ; t_{2}(59)=2.94, p<.01\right)$. In contrast, there were no similar reliable effects for the compound words and the 
phrases. Thus, there was a parafoveal-on-foveal influence of the second character on first fixations on the first character for monomorphemic words, but no similar effect for the compound words and phrases $(t \mathrm{~s}<1.43)$.

Gaze Duration. For gaze duration, there was a significant main effect of preview type, $F_{1}(1,47)=7.17, M S_{e}=1,289, p<.01 ; F_{2}(1,59)=5.36, M S_{e}=2,079, p<.05$, gaze durations were longer for the dissimilar previews $(254 \mathrm{~ms})$ than for the identical previews $(242 \mathrm{~ms})$. There was no significant main effect of target type $(F \mathrm{~s}<1.22)$ on gaze durations indicating that regardless of the type of target character, gaze durations for the first character were similar. Once again, however, there was a significant target type by preview interaction $\left(F_{1}(2,94)=3.97, M S_{e}=1,488, p<.05\right.$; $\left.F_{2}(2,118)=6.70, M S_{e}=1,812, p<.01\right)$. As for the first fixation duration measure, there was a significant difference between identical and dissimilar preview conditions for monomorphemic word $(25 \mathrm{~ms}), t_{1}(47)=3.76, p<.001 ; t_{2}(59)=3.57, p<.01$. There was no significant difference for the compound words $(t \mathrm{~s}<1)$. The preview effect for the phrases $(15 \mathrm{~ms})$ approached, but did not attain, significance, $t_{1}(47)=$ $1.69, p=.10 ; t_{2}(59)=1.88, p=.07$. These results indicate that for gaze durations on the first character of the target character pair, robust parafoveal-on-foveal effects occurred when that character was part of a monomorphemic word. The extent to which similar effects occurred when the first character was part of a compound word was greatly reduced (and not reliable), and when the first character of the target was part of a phrase, there was no evidence of parafoveal-on-foveal effects. Thus, it appears that whilst the first character is being fixated the lexical characteristics of a 
two character target string fundamentally determine the extent to which the second character is pre-processed. Parafoveal-on-foveal effects occurred most clearly when the first character signaled strongly that the adjacent character to the right was likely to be a constituent part of the word unit.

Additional Analyses. For completeness, we also computed the regression path time and the word skipping rates for the first character (see Table 2). Regression path time was defined as the sum of all fixations from the first fixation on the first character of the target until a fixation to the right of that character. In fact, readers made very few regressions from the first character of the target, and consequently, the mean regression path time and preview effects were very similar to those for gaze duration. There was a significant main effect of preview type, $F_{1}(1,47)=7.12, M S_{e}$ $=4,658, p<.01 ; F_{2}(1,59)=11.75, M S_{e}=5,594, p<.01$, with regression path time being longer for the dissimilar previews $(293 \mathrm{~ms})$ than for the identical previews (272 $\mathrm{ms})$. There was no significant main effect of target type $(F \mathrm{~s}<1)$, indicating regression path time on the first character was similar regardless of the nature of the target character string. Finally, the target type by preview type interaction was reliable by subjects, but not items $\left(F_{1}(2,94)=3.78, M S_{e}=5,153, p<.05 ; F_{2}(2,118)=\right.$ 2.33, $\left.M S_{e}=6,903, p=.09\right)$. The means patterned very similarly to those obtained for the first fixations and gaze durations with the differences between identical and dissimilar previews for monomorphemic words being reliable $(41 \mathrm{~ms}), t_{1}(47)=2.68$, $p<.01 ; t_{2}(59)=3.67, p<.01$, whilst the same effects for compound words and phrases were not reliable ( $t \mathrm{~s}<1.77$ ). Again, these results support the claim that the 
nature of target character pair modulated the extent to which the second character influenced fixation durations on the first character. Turning to the first character skipping probability, there was only a main effect of target type $\left(F_{1}(2,94)=6.64, M S_{e}\right.$ $=.03, p<.01 ; F_{2}(2,118)=6.83, M S_{e}=.03, p<.01$; all other $\left.F \mathrm{~S}<1\right)$. This effect is slightly inconsistent with the patterns for the other measures in that the skipping rate was higher for compound words $(.38)$ than monomorphemic words $\left(.30 ; t_{1}(47)=3.28\right.$, $\left.p<.01 ; t_{2}(59)=3.33, p<.01\right)$ and phrases $\left(.32 ; t_{1}(47)=2.96, p<.01 ; t_{2}(59)=3.03, p\right.$ $<.01)$. We have no ready explanation for this effect. It cannot be the case that readers skipped the first character more often when it formed a word, otherwise we would have observed similar skipping rates for the compound and phrase target character pairs. This was not the case. Suffice it to say, there is a paucity of experimental studies investigating character and word skipping in Chinese and this area requires future research.

\section{Eye fixation measures for the second constituent}

The eye fixation measures for the second constituent index the processing of the second constituent after the parafoveal preview has been changed to its intended form. These measures reflect how the target character pair as a whole was processed in relation to the previews (see Table 2).

First Fixation Duration. For the first fixation duration, there was a marginal main effect of target type, $F_{1}(2,94)=2.55, M S_{e}=1,982, p=.08 ; F_{2}(2,118)=2.47$, $M S_{e}=1,763, p=.09$. The means patterned such that the first fixation duration was 
numerically, but not reliably, shorter for monomorphemic words $(252 \mathrm{~ms})$ than compound words $\left(262 \mathrm{~ms} ; t_{1}(47)=1.74, p=.09 ; t_{2}(47)=1.72, p=.09\right)$ and reliably shorter than phrases $\left(266 \mathrm{~ms} ; t_{1}(47)=1.99, p=.05 ; t_{2}(47)=2.15, p<.05\right)$. Meanwhile there was no difference $(t \mathrm{~s}<1)$ between compound words and phrases. The pattern of differences suggests that the increased parafoveal processing observed for the first character measures resulted in more efficient processing of the second constituent when it was first fixated. There was a significant main effect of preview type, $F_{1}(1,47)=45.83, M S_{e}=3,163, p<.001 ; F_{2}(1,59)=79.03, M S_{e}=2,446, p$ $<.001$, with longer first fixation durations for the dissimilar previews $(282 \mathrm{~ms})$ than for the identical previews $(237 \mathrm{~ms})$. This is unsurprising and reflects the basic preview effect (e.g., Rayner, 1975). There was no significant target type by preview type interaction $\left(F_{\mathrm{S}}<1\right)$, indicating that any beneficial effects of the preview of the second character did not occur in processing times for that character when it was directly fixated. Thus, it appears that the parafoveal-on-foveal effects that occurred for the first character were short lived, failing to carry over and influence the duration of the first fixation on the second character of the target character pair.

Gaze Duration. For gaze duration, the effects were very similar to those for the first fixation duration data. There was a significant main effect of target type, $F_{1}(2,94)=5.74, M S_{e}=2,524, p<.01 ; F_{2}(2,118)=3.37, M S_{e}=3,688, p<.05$. The gaze duration was shorter for monomorphemic words $(271 \mathrm{~ms})$ than compound words $\left(292 \mathrm{~ms} ; t_{1}(47)=2.85, p<.01 ; t_{2}(59)=2.48, p<.05\right)$ and phrases $\left(292 \mathrm{~ms} ; t_{1}(47)=\right.$ $\left.2.81, p<.01 ; t_{2}(59)=2.24, p<.05\right)$; meanwhile there was no significant difference 
$(t \mathrm{~s}<1)$ between the compound words and phrases. Once more, this probably reflects the extent to which the second character was processed prior to its fixation. There was also a significant main effect of preview type, $F_{1}(1,47)=71.11, M S_{e}=4,193, p$ $<.001 ; F_{2}(1,59)=121.13, M S_{e}=3,265, p<.001$; gaze durations for the dissimilar previews were longer than for the identical previews. There was no significant interaction $(F \mathrm{~S}<1)$, indicating again that preview benefit effects were similar for all three target types.

Additional Analyses. As for the first character of the target, for completeness, we also computed regression path time and word skipping rates for the second character of the target (see Table 2). For regression path time, there was a main effect of target type (significant by subjects and very close by items), $F_{1}(2,94)=7.02, M S_{e}=6,928, p$ $<.01 ; F_{2}(2,116)=2.93, M S_{e}=15,301, p=.06$. The regression path time was shorter for monomorphemic words $(349 \mathrm{~ms})$ than compound words $\left(384 \mathrm{~ms} ; t_{1}(47)=2.74, p\right.$ $\left.<.01 ; t_{2}(59)=2.12, p<.05\right)$ and phrases $\left(392 \mathrm{~ms} ; t_{1}(47)=3.51, p<.01 ; t_{2}(59)=2.41\right.$, $p<.05)$. There was no significant difference $(t \mathrm{~s}<1)$ between the compound word and phrase. Clearly, later processing of the second character of the target was more efficient for monomorphemic words compared with compound words and phrases. As with the other measures, presumably this also reflects the facilitatory parafoveal effects for monomorphemic words relative to the compound words and phrases observed for the earlier measures.

There was also a significant main effect of preview type, $F_{1}(1,47)=65.62, M S_{e}=$ $14,907, p<.001 ; F_{2}(1,58)=108.56, M S_{e}=12,446, p<.001$, with regression path 
time being longer for the dissimilar relative to the identical previews. Again, this is an unsurprising finding reflecting the availability of useful parafoveal information. There was no significant target type by preview type interaction $(F \mathrm{~s}<1)$ indicating that any beneficial effects of the preview of the second character were no longer observable as the second character was processed.

For the probability of skipping over the second constituent, there was only a significant main effect of preview, $F_{1}(1,47)=50.97, M S_{e}=.03, p<.001 ; F_{2}(1,59)=$ $68.54, M S_{e}=.03, p<.001$, and unsurprisingly, this reflected the fact that the skipping rate was higher when the preview was identical compared with when it was a nonsense character.

\section{Eye fixation measures for the whole target character pair}

For the whole target character pair we report only the gaze duration data since single and first fixations were necessarily on one or other (but not both) of the constituent characters (see Table 2). For the gaze duration, there was a significant main effect of preview type, $F_{1}(1,47)=101.31, M S_{e}=9,604, p<.001 ; F_{2}(1,59)=$ 144.82, $M S_{e}=8,149, p<.001$. Gaze durations for the dissimilar previews were longer than for the identical previews. There was no significant main effect of target type, nor was there a significant target type by preview type interaction $\left(F_{\mathrm{S}}<1\right)$, again indicating that these effects were quite short lived.

First Pass Fixation Count. For the first pass fixation count, there was no significant main effect of preview type and target type, nor was there a significant 
target type by preview type interaction $\left(F_{\mathrm{S}}<1.83\right)$, indicating that the first fixation pass count was similar for different kinds of target word.

Additional Analyses. For the whole two character target we also computed regression path time and skipping rates (see Table 3). For regression path time, there was a significant main effect of preview type, $F_{1}(1,47)=53.04, M S_{e}=26,902, p$ $<.001 ; F_{2}(1,59)=84.32, M S_{e}=21,605, p<.001$, with longer times for dissimilar than identical previews. There was no significant main effect of target type, nor was there an interaction between preview and target type $\left(F_{\mathrm{S}}<1\right)$. We also found that there were no reliable effects on the probability of skipping over the target character pair $(F \mathrm{~s}<1.14)$

\section{Discussion}

From studies investigating reading of spaced alphabetic languages, it has been demonstrated that both word spacing information within sentences and the morphological structure of a word can determine the extent to which its constituents are processed serially or in parallel. In our study, we directly investigated two questions associated with this issue in relation to Chinese reading: First, whether the lexical status (word or phrase) of a pair of Chinese characters influenced whether they were processed serially or in parallel. Secondly, when the character pair formed a word, we asked whether the morphological characteristics of that word affected whether the pair of characters was processed serially or in parallel. These questions are novel, and particularly interesting since Chinese is a dense character based, 
unspaced language in which there is no overt demarcation of word boundaries, and indeed, where there can often be ambiguity as to the characters that comprise a word.

For target character pairs that comprised monomorphemic words, we found that when the parafoveal preview of the second character of the pair was a nonsense character, fixations on the first character were longer than when the preview of the second character was the character itself. This indicates that the character to the right of the fixated character was processed prior to its direct fixation, a reliable parafoveal-on-foveal effect. Note, however, that there was no similar effect for target character pairs that were compound words or phrases. This finding suggests that the two characters of monomorphemic words were processed in parallel, whereas when the target character pair comprised a compound word or a phrase, they were processed serially. This finding is very consistent with the results of Drieghe, Pollatsek, Juhasz and Rayner (2010), who also found within word parafoveal-on-foveal effects for monomorphemic words but not for compound words. The present result indicates that the morphological structure of a word, or alternatively, the predictability of the second character on the basis of the first character fundamentally determines how the word is processed during reading.

Let us briefly consider the implications of this result in some more detail. It is tempting to conclude that Chinese readers preprocess the second character of the target pair to a deeper, probably lexical, level when the first character belongs to a monomorphemic word, than is the case when they are fixating the first character of a compound word or a phrase. If this view was correct, then the difference in the 
"lexical depth" to which the second character is processed prior to fixation (e.g., to a shallow orthographic level, or instead to a higher morphemic or even lexical level) could cause the effects observed in fixations on the first character. However, this conclusion may go slightly beyond the data. Arguably, a more conservative view may be that when the first character of the target indicates that it is part of a monomorphemic, two character word, then this simply licenses processing of the adjacent character to the right. Under such circumstances the oddity of the orthographic information of the nonsense character available in the preview might result in the increased fixation observed on the first character of the pair. In contrast, for compound words and phrases, similar processing of the parafoveal character may not be licensed since the first character of the target may form a word in its own right, and therefore does not require the appendage of the second character to form a recognizable lexical unit.

Another noteworthy point in relation to these results is that since the preview effect that arose for fixations on the second character of the target was identical for all three types or target character pairs, then the differential parafoveal-on-foveal effects that occurred for the monomorphemic target words cannot have arisen due to our manipulation being ineffective for the compound word and phrase stimuli. Clearly, the preview manipulation was very effective for all three types of stimuli, however, the time course of its influence was different for monomorphemic words relative to compound words and phrases. Overall, then, the pattern of parafoveal-on-foveal effects, along with the preview effects for the different types of target word are very 
clear and quite simple to interpret.

Having said this, there is an aspect of the data that may initially appear surprising. The data for the target character pairs that were compound words patterned very similarly to the data for target character pairs that were phrases, and both of these patterned differently from the data for the monomorphemic target characters. Compound words, like monomorphemic words, have lexical entries associated with them, whereas phrases do not. Indeed, the meaning of phrases is generally considered to be computed online on the basis of the individual constituent words as opposed to accessed directly from an entry in the mental lexicon. Thus, it may have been reasonable to expect that the data for the compound target words would pattern similarly to the data for the monomorphemic words, and differently to the data for the phrases. This was clearly not the case.

Why, then, do the data for the compound words pattern similarly to those for the phrases, and dissimilarly to those for the monomorphemic words? In our experiment we were extremely careful to select our target stimuli strictly on the basis of their dictionary categorization. We did this for the monomorphemic words and the compound words, and we also ensured that none of the phrases appeared as an entry in the dictionary for a single word. Thus, in terms of their formal linguistic characterization, our stimuli were clearly from three distinct categories. However, whilst the formal categorization of whether a pair of Chinese characters do, or do not, comprise a word is consistent across different Chinese dictionaries (e.g., Modern Chinese Word Dictionary, 2005; The Chinese Daily Word Frequency Dictionary, 
1998; The Modern Chinese Word Frequency Dictionary, 1990), it is not necessarily the case that similar consistency applies across different Chinese readers. As we mentioned earlier, Chinese readers do not always agree on those characters that comprise the words within a sentence, and the question of what constitutes a "psychologically real" word in Chinese readers has been considered within the literature (e.g., Hoosain, 1992; Li, Rayner \& Cave, 2009).

Given this, we were keen to explore whether individual readers' categorizations of character strings as words or phrases might have more of a bearing on how they process them as they read normally. To do this, we first conducted an off-line assessment of a group of participants' judgements about the lexical status (word or phrase) of our target character pairs in just the compound word and the phrase conditions when they appeared in their sentential contexts. Two lists of experimental sentences on sheets of paper were prepared. The experimental sentences were identical to those that we had used in the eye movement experiment, and of course included the target compound words and phrases. The type of target character pair was counterbalanced across lists using a Latin square design such that half the stimuli contained a phrase target pair, and half a compound word. Each sentence frame only appeared once in each list. Thirty-four participants took part in the assessment, with 17 assigned to each list. Participants were required to mark with a "/" all of the word boundaries in the sentence. If participants judged the target character pair to be one word, we provided a score of 1; if they judged the target character pair to be two words, we provided a score of 0 . 
The analyses showed that for target character pairs that were compound words, participants rated them as compound words $74 \%(\mathrm{SD}=.16)$ of the time. For the target character pairs that were adjective noun phrases, participants rated them as compound words $45 \%(\mathrm{SD}=.15)$ of the time. A $t$-test confirmed that the compound target character pairs were correctly categorized as a compound word significantly more often than a phrase was categorized as a single word, $t_{1}(33)=8.08, p<.001 ; t_{2}(59)=$ $11.60, p<.001$, (and note that by implication, the counterpart effect for phrase and compound word categorizations respectively, was also true). These results are informative in three respects. First, they indicate that our characterization of the target character pairs as either compound words or phrases was broadly correct. There was a significant bias to categorize two character phrases as phrases, and not as compound words, and a bias to categorize two character compound words as compound words and not as phrases. Thus, the processing biases that we anticipated would exist for our stimuli did exist. The second conclusion the results inform, however, qualifies the first. It is very clear that although there were biases in processing that we predicted, these were far from categorical. Readers quite often categorized the two character phrases as compound words, as well as categorizing the two character compound words as phrases. This finding reinforces our claim that there is ambiguity amongst Chinese readers as to the characters that do and do not comprise words. Finally, the data indicate that the propensity to miscategorize a two character target was much greater for the phrases than was the case for the compound words. Thus, there is an overall bias amongst Chinese readers when faced with a 
decision as to whether an ambiguous two character string is one or two words, to commit to it being one word. Furthermore, one might cautiously conclude that this could arise due to the predominance of two character words in the Chinese language.

Extending these analyses, based on the categorization data, we were able to identify a subset of our stimuli that participants most often judged to be phrases, along with another subset that participants most often judged to be compound words. We selected 21 items from our stimulus list for which more than $82 \%$ participants in the off-line rating study indicated that the target character pair was a single word. We also selected another 21 items for which fewer than $36 \%$ of participants judged that the target character pair was a single word (i.e., they categorized the character pair as representing two words). We then examined whether there were differential effects in the eye movement data for these two groups of stimuli, given that these were the best representative instances of the categories we used in our experiment. We predicted that robust preview effects would occur, but that there would be no interaction between the type of target character pair and the preview. That is, consistent with the main findings reported earlier, we expected the eye movement data to reflect a similar degree of parafoveal processing for both the phrases and the compound words. Indeed, this was exactly what we found.

For the fixation durations on the first constituent of the target character pair, there was neither a significant main effect of word type and preview type, nor a word type by preview type interaction $(F \mathbf{s}<1)$. The results did not provide any evidence for parafoveal-on-foveal effects for compound words or phrases. 
We also examined the first fixation duration on the second constituent of the target character pair. There was a significant main effect of preview type, $F_{2}(1,20)=$ 23.95, $M S_{e}=1,774, p<.001$, with a longer first fixation duration for the dissimilar previews $(287 \mathrm{~ms})$ than for the identical previews $(242 \mathrm{~ms})$. There was no significant main effect of word type, nor a word type by preview type interaction $\left(F_{\mathbf{s}}<1\right)$. There was an identical preview benefit for both compound words and phrases (45ms). The patterns for gaze durations on the second constituent for the target character pair were identical.

In summary, we conducted a boundary technique eye movement experiment to investigate preview effects in relation to the constituent characters of two character Chinese monomorphemic and compound words, as well as phrases. This experiment was similar to an experiment in English reported by Drieghe et al. We were particularly interested to understand the extent to which readers processed the second character of the target character pair when they were fixating the first character, and whether any such processing was modulated by the type of target character pair being processed. We found that parafoveal-on-foveal effects of the adjacent character only occurred when the target character pair formed a monomorphemic word, not when it was a compound word or a phrase. There was clear modulation of parafoveal processing contingent on the linguistic category of the target character pair. We interpret this effect as indicating that when the first character of a two character Chinese string signifies that an adjacent partner character is required in order to form a meaningful word either due to morphological or probabilistic characteristics, this 
licences pre-processing of that adjacent character prior to its direct fixation. Thus, readers are sensitive to information about the types of the characters that comprise two character words compared with phrases, and also use such information to discriminate between different types of words. Furthermore, they use this information on-line to moderate parafoveal processing of upcoming characters. Finally, we presented evidence to suggest that although formal linguistic (dictionary) definitions of words capture categorical differences between words in Chinese, Chinese readers are often divided in their judgments regarding what comprises a word. 


\section{References}

Balota, D. A., \& Rayner, K. (1991). Word recognition processes in foveal and parafoveal vision: The range of influence of lexical variables. In D. Besner \& G. W. Humphreys (Eds.), Basic processes in reading. Hillsdale, NJ:Erlbaum.

Cai, Q., \& Brysbaert, M. (2010). SUBTLEX-CH: Chinese Word and Character Frequencies Based on Film Subtitles. Plos ONE, 5(6), e10729.

Cui, 1., Yan, G., Bai, X., Hyönä, J., Wang, S., \& Liversedge, P. S. (2012). Processing of compound-word characters in reading Chinese: An eye-movement-contingent display change study. Quarterly Journal of Experimental Psychology.

Drieghe, D. (2011). Parafoveal-on-foveal effects on eye movements during reading, pp. 839-855. In The Oxford Handbook on Eye Movements, Liversedge, S.P., Gilchrist, I.D. \& Everling, S. (Eds.), Oxford University Press, Oxford, UK.

Drieghe, D., Pollatsek, A., Juhasz, B.J., \& Rayner, K. (2010). Parafoveal processing during reading is reduced across a morphological boundary. Cognition, $116,136-142$.

Engbert, R., Nuthmann, A., Richter, E.D., \& Kliegl, R. (2005). SWIFT: A dynamical model of saccade generation during reading. Psychological Review, 112, $777-813$.

Hoosain, R. (1992). Psychological Reality of the word in Chinese. In H.-C. Chen \& O. J.L. Tzeng. (Eds.), Language processing in Chinese (pp. 111-130). Amsterdam, Netherlands: North-Holland. 
Hyönä, J., Bertram, R., \& Pollatsek, A. (2004). Are long compound words identified serially via their constituents? Evidence from an eye-movement-contingent display change study. Memory \& Cognition, 32, 523-532.

Juhasz, B.J., Pollatsek, A., Hyönä, J., Drieghe, D., \& Rayner, K. (2009). Parafoveal processing within and between words. Quarterly Journal of Experimental Psychology, 62, 1356-1376.

Kennedy, A. (2000). Parafoveal processing in word recognition. Quarterly Journal of Experimental Psychology, 53, 429-455.

Li, X.S., Rayner, K., \& Cave, K. (2009). On the segmentation of Chinese words during reading. Cognitive Psychology, 58, 525-552.

Murray, W.S. (1998). Parafoveal pragmatics. In G. Underwood (Ed.), Eye guidance in reading and scene perception (pp. 181-200). Oxford: Elsevier.

Rayner, K. (1975). The perceptual span and peripheral cues during reading. Cognitive Psychology, 7, 65-81.

Rayner, K., Well, A.D., Pollatsek, A., \& Bertera, J.H. (1982). The availability of useful information to the right of fixation in reading. Perception \& Psychophysics, 31, $537-550$.

Rayner, K. (1998). Eye movements in reading and information processing: 20 years of research.Psychological Bulletin, 124, 372-422.

Reichle, E.D., Pollatsek, A., Fisher, D.L., \& Rayner, K. (1998). Toward a model of eye movement control in reading. Psychological Review, 105, 125-157.

Reichle, E.D., Rayner, K., \& Pollatsek, A. (2003). The E-Z Reader model of eye 
movement control in reading: Comparison to other models. Behavioral and Brain Sciences, 26, 445-476.

Schotter, E.R., Angele, B., \& Rayner, K. (2012). Parafoveal Processing in Reading. Attention, Perception, \& Psychophysics, 74, 5-35.

Wang, S., Tong, X., Yang, J., \& Leng, Y. (2009). Semantic codes are obtained before word fixation in Chinese sentence reading: Evidence from eye-movements. Acta Psychologica Sinica, 41, 220-232.

Yan, M., Richter, E.D., Shu. H., \& Kliegl, R. (2009). Readers of Chinese extract semantic information from parafoveal words. Psychonomic Bulletin \& Review, 16, $561-566$.

Yang, J., Wang, S., Xu, Y., \& Rayner, K. (2009). Do Chinese readers obtain preview benefit from character $\mathrm{n}+2$ ? Evidence from eye movements. Journal of Experimental Psychology: Human Perception and Performance, 35, 1192-1204.

Yen, M-H., Tsai, J-L., Tzeng, O.J.I., \& Hung, D.L. (2008). Eye movements and parafoveal word processing in reading Chinese. Memory \& Cognition, 36, 1033 $-1045$. 


\section{Author Note}

We would like to thank two anonymous reviewers for helpful comments on an earlier draft of this paper. The research described in this article along with the write up were supported by: a grant from the Social Science Foundation of China (10BYY029) for the third author; Key Research topics of the Ministry of Education Award for the $11^{\text {th }}$ Five-year Plan of National Science of Education (DBA090290) and National Science Foundation of China (30870781) for the fourth author; ESRC awards (RES-000-22-4128 \& ES/I032398/1) and a Leverhulme Trust Grant (No F/00 180/AN) to the sixth author. The authors also acknowledge support from the China Scholarship Council. 


\section{Figure Legend}

Figure 1. Example Chinese stimuli for the different experimental conditions.

The target characters were monomorphemic words, compound words and adjective noun phrases. These appear in bold in the sentences (though they appeared normally in the experiment). The location of the invisible boundary is indicated by the vertical line. The preview was either an identity preview (e.g., 瑰 or 塔) or a dissimilar

pseudocharacter (e.g., 捝), and this was initially displayed in place of the second character of the target character pair. When the readers' eyes crossed the invisible boundary location, the preview was replaced by the target character. 
Figure 1.

(1) Monomorphemic word

文章中描写玟师鬼的语句很美。

文章中描写牫捝的语句很美。

The description of rose is very beautiful in the paper.

(2) Compound word

文章中描写灯塔的语句很美。

文章中描写灯捝的语句很美。

The description of beacon is very beautiful in the paper.

(3) Adjective noun word combination

文章中描写斜塔的语句很美。

文章中描写斜说的语句很美。

The description of leaning tower is very beautiful in the paper. 
Table 1.

Lexical-Statistical Properties for the Monomorphemic Words, Compound Words and Phrase.

\begin{tabular}{lccc}
\hline & Monomorphemic & Compound & Phrase \\
\hline Frequency of first character & 43 & 249 & 249 \\
Strokes of first character & 10.8 & 8.48 & 9.07 \\
Frequency of second character & 61 & 586 & 586 \\
Strokes of second character & 10.2 & 7.58 & 7.58 \\
Word frequency & 6.03 & 3.42 & - \\
\hline
\end{tabular}

Note: Word frequency is measured as a words-per-million figure using the Chinese Daily Word Frequency Dictionary (1998). Character frequency is measured as a characters-per-million figure (Cai and Brysbaert, 2010). 
Table 2.

Eye Fixation Measures (in ms) for the Target Character Pair, as a Function of Target Type and Preview Type.

\begin{tabular}{|c|c|c|c|c|c|c|c|c|c|c|}
\hline & & \multicolumn{3}{|c|}{ Monomorphemic } & \multicolumn{3}{|c|}{ Compound } & \multicolumn{3}{|c|}{ Phrase } \\
\hline & & $\mathrm{I}$ & $\mathrm{D}$ & $\mathrm{PE}$ & $\mathrm{I}$ & $\mathrm{D}$ & $\mathrm{PE}$ & $\mathrm{I}$ & $\mathrm{D}$ & PE \\
\hline & FFD & 229 & 246 & 17 & 233 & 226 & -7 & 231 & 242 & 11 \\
\hline First character of the & GAZE & 240 & 265 & 25 & 246 & 240 & -6 & 241 & 256 & 15 \\
\hline \multirow[t]{3}{*}{ target character pair } & $\mathrm{RPT}$ & 270 & 311 & 41 & 274 & 281 & 7 & 272 & 288 & 16 \\
\hline & SKIP & .30 & .31 & .01 & .38 & .39 & .01 & .32 & .33 & .01 \\
\hline & FFD & 225 & 278 & 53 & 241 & 283 & 42 & 246 & 286 & 40 \\
\hline Second character of the & GAZE & 235 & 306 & 72 & 260 & 323 & 63 & 263 & 321 & 58 \\
\hline \multirow[t]{2}{*}{ target character pair } & $\mathrm{RPT}$ & 292 & 406 & 114 & 322 & 445 & 123 & 336 & 448 & 112 \\
\hline & SKIP & .40 & .23 & -.17 & .38 & .22 & -.16 & .39 & .25 & -.14 \\
\hline
\end{tabular}




\begin{tabular}{lcccccccccccccc}
\hline & GAZE & 330 & 459 & 129 & 344 & 450 & 105 & 346 & 461 & 115 \\
& & RPT & 413 & 561 & 149 & 417 & 567 & 150 & 440 & 563 & 124 \\
Whole target character pair & SKIP & .06 & .05 & -.01 & .07 & .06 & -.01 & .06 & .05 & -.01 \\
& & & & & & & & & & & & \\
& FPFC & 1.63 & 1.68 & .05 & 1.64 & 1.64 & .001 & 1.62 & 1.73 & .11 \\
\hline
\end{tabular}

Note: FFD = first fixation duration; GAZE = gaze duration; RPT = regression path time; Skip = probability of skipping; FPFC $=$ first pass fixation count;

$\mathrm{I}=$ Identical; $\mathrm{D}=$ Dissimilar; PE $=$ Preview Effects $(\mathrm{D}-\mathrm{I})$. 